\title{
Congenital syphilis in The Netherlands: cause and parental characteristics
}

\author{
J M BOOT,* H E MENKE, $\dagger \mathrm{R} \mathrm{V} \mathrm{W} \mathrm{vaN} \mathrm{EIJK,} \dagger$ A P ORANJE, * E STOLZ* \\ From the *Department of Dermatology, Rotterdam University Hospital Dijkzigt, Rotterdam, and the \\ †Laboratory for Bacteriology, National Institute of Public Health and Environmental Protection, Bilthoven, The \\ Netherlands
}

SUMMARY During 1982-5 the 19S (IgM) fluorescent treponemal antibody absorption (19S (IgM) FTA-ABS) test gave positive results in 19 children. The parental histories were analysed. As five of the children were adopted, 14 pregnancies were evaluated.

Mothers of foreign origin and extramarital pregnancies were found to be over-represented. Of 13 women who attended for pregnancy checkup, three were not serologically screened for syphilis. In four the infection had developed late in the course of pregnancy. In at least four treatment had not been given or had been inadequate or too late. At least two had positive 19S (IgM) FTA-ABS test results that did not indicate congenital syphilis. The possibility of false positive 19S (IgM) FTA-ABS test results is pointed out.

As the male sexual partners of four of the 14 mothers had presented elsewhere with early syphilis at the time of their partner's pregnancy, adequate contact tracing appears to be important to prevent congenital syphilis in future.

Congenital syphilis still occurs in The Netherlands. This is distressing because, as a part of pregnancy check up, every woman is supposed to be tested for the presence of anticardiolipin and antitreponemal antibodies. If the results of these tests in combination with findings on clinical investigation indicate the presence of syphilis, and timely and adequate treatment is given, congenital syphilis is preventable.

There is no consensus about the diagnosis of congenital syphilis. ${ }^{1}$ The diagnosis is certain if the pathogen, Treponema pallidum, is shown in body fluids by dark field microscopy, by methods such as immunofluorescence tests that detect antigen, or in tissue biopsy specimens (usually of skin) stained with silver. Serological indications of congenital syphilis are a fourfold rise in the titre at which the Venereal Disease Research Laboratory (VDRL) test result is positive or a positive 19S IgM fluorescent treponemal antibody absorption (FTA-ABS) test result, or both. Other indications may be found in clinical signs, such

Address for reprints: Professor E Stolz, Department of Dermatovenereology, Rotterdam University Hospital Dijkzigt, Rotterdam, The Netherlands

Accepted for publication 26 April 1988 as haemorrhagic rhinitis, skin lesions, bone lesions, hepatosplenomegaly, or anaemia. Radiography of the long bones and tests of the cerebrospinal fluid (CSF) may show abnormal findings.

The exact number of cases of congenital syphilis in The Netherlands is not known, although the disease has to be notified to the chief public health inspector of the Ministry of Health. Five cases were registered in 1982-5, but in the same period the department of venereology of the National Institute of Public Health and Environmental Protection found positive 19S IgM FTA-ABS test results in serum samples from 19 children. We analysed the case histories of these 19 children and their parents to see whether the positive 19S IgM FTA-ABS test results indicated that the children had congenital syphilis. We tried to ascertain why certain pregnancies had resulted in congenital syphilis. On the basis of these findings, we recommend ways of further reducing the number of children with congenital syphilis in the future.

\section{Patients and methods}

Serum samples from neonates with suspected congenital syphilis were sent by various clinics in The 
Netherlands to the National Institute of Public Health and Environmental Protection for confirmation testing. All specimens were tested with the $T$ pallidum haemagglutination assay (TPHA) and the FTA-ABS, VDRL, and 19S (IgM) FTA-ABS tests. IgM was separated from serum by gel filtration chromatography using high performance liquid chromatography equipment.

During 1982-5 positive 19S (IgM) FTA-ABS test results were found in 19 children. The names of the patients and their attending doctors were obtained from the forms requesting the tests. Each attending doctor was asked to complete a questionnaire concerning the history of the mother, her sexual partner, and the child. If possible, the child's mother was approached for additional information. All data were processed anonymously.

\section{Results}

We evaluated 19 children with positive 19S (IgM) FTA-ABS test results. The table lists the causes in relation to serological syphilis screening during pregnancy. Serological screening for syphilis was performed during 10 pregnancies, three women were not screened, and we did not known whether or not the other six had been screened as five of the children concerned had been adopted from abroad.

The first column of the table shows that infection developed late in the course of pregnancy in four women, three of whom (cases 1, 2, and 3) had previously had negative serological screening results. We did not know whether or not the fourth (case 4) had been screened, but her husband developed primary syphilis in week 37 of her pregnancy and she developed secondary syphilis seven weeks after parturition, by which time the child had clinical signs of congenital syphilis.

Early syphilis was also diagnosed in the sexual partner of one woman (case 1) at the time of her pregnancy, but contact tracing was not undertaken until after parturition. The serology of the child was studied; after three months serological test results were as follows: TPHA positive, FTA-ABS strongly positive, VDRL positive at a titre of $1 / 128$, and $19 \mathrm{~S}$ (IgM) FTA-ABS strongly positive. The child also had clinical signs of congenital syphilis and was treated.

One of the three women with a negative screening result at the start of the pregnancy (case 3) was a prostitute addicted to heroin, who could therefore be considered as being at high risk for contracting syphilis.

Three women (cases 5, 6, and 7) were not screened for syphilis. The child of one (case 5) developed clinical signs of congenital syphilis four weeks after parturition. Both parents had weakly positive TPHA results and the VDRL test result was positive at a titre of $1 / 4$. Both parents and the child were treated. In one (case 6) no routine screening was performed because normal pregnancies had occurred 17 and 18 years previously. In the third (case 7) a previous pregnancy had also been uneventful. During the seventh month of her second pregnancy, however, her sexual partner contracted primary syphilis, but did not mention the mother as being a sexual contact. Three weeks after birth the child showed clinical signs of congenital syphilis and was treated.

The third column of the table shows that for at least four women treatment was omitted, inadequate, or too late. Two (cases 8 and 9) were not treated despite positive syphilis serology test results found on screening in week 14 of pregnancy. One (case 8) had weakly positive results in the TPHA and the FTA-ABS test, and the VDRL test result was positive at a titre of $1 / 32$, but no action was taken. Three months after birth the child presented with clinical signs consistent with syphilis; mother and child were treated. The serology test results of her sexual partner were negative; there was no information about other partners. The other untreated woman (case 9) had weakly positive results in the TPHA and the FTAABS test and a negative VDRL test result in week 14 of pregnancy. These results may indicate treated syphilis or untreated late syphilis. Although the mother had never been treated for syphilis, however, no treatment was given until after parturition. The child was

Table Evaluation of 19S (IgM) fluorescent treponemal antibody absorption (FTA-ABS) positive test results in 19 children (1982-5)

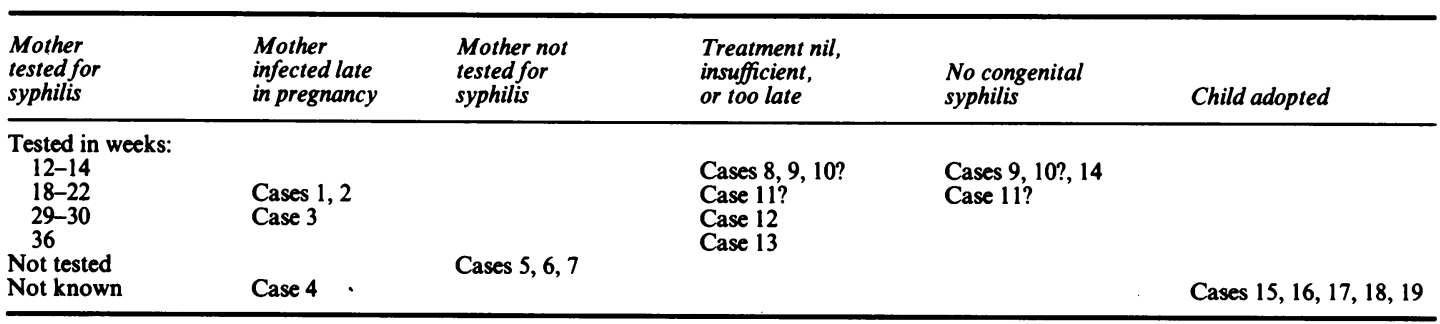


serologically tested. The umbilical cord blood gave weakly positive results in the TPHA and the FTAABS and 19S (IgM) FTA-ABS tests and negative VDRL test results. The tests were repeated. The 19S (IgM) FTA-ABS test gave negative results after the first week and remained negative: six months after birth the TPHA and the FTA-ABS test also gave negative results. The child did not develop congenital syphilis. The positive 19S (IgM) FTA-ABS test result did not indicate congenital syphilis. The fact that the child did not develop the disease does not, however, justify not treating the mother during her pregnancy.

It was not clear whether the child of one woman (case 10) developed congenital syphilis. The woman's sexual partner had been treated for primary syphilis five years previously and had been treated again six months later in view of a positive VDRL test result at a titre of $1 / 64$. Three months before conception his VDRL test result was negative, his TPHA result positive, and the CSF was normal except for a positive TPHA result. During her pregnancy check up in week 12 the woman had a weakly positive FTA-ABS test result and positive results in the TPHA at a titre of $1 / 20480$ and in the VDRL at a titre of $1 / 8$. She was treated with $2.4 \mathrm{MIU}$ benzathine penicillin $\mathrm{G}$ three times in two weeks. As the VDRL test result remained positive at a titre of $1 / 8$, this treatment was repeated in week 24-25 of pregnancy. After parturition the VDRL test result remained positive at a titre of $1 / 8$. The woman refused a lumbar puncture for CSF analysis. The serology results of the child seven days after birth were as follows: TPHA positive, FTA-ABS strongly positive, VDRL positive at a titre of $1 / 2$, 19S (IgM) FTA-ABS weakly positive. Apart from the positive 19S (IgM) FTA-ABS result, there were no indications of the presence of syphilis. The child was treated for possible congenital syphilis. The presence of antitreponemal IgM may have been because the mother had been inadequately treated or reinfected. Another possibility is that it was not related to congenital syphilis. As the titre showing positivity in the VDRL test of the mother showed no interim increase, her reinfection after week 24 was unlikely.

A similar patient (case 11) was a woman who showed a weak positive result in the FTA-ABS test and positive results in the TPHA at a titre of $1 / 80$ and in the VDRL test at a titre of $1 / 8$ in the fourth month of pregnancy. Her sexual partner was found to have been treated for early latent syphilis two months previously, but he had not mentioned the woman as being a sexual contact. She was treated in the fourth month with 0.6 MIU procaine benzyl penicillin eight times and 1.2 MIU benzathine benzyl penicillin twice. Three months after treatment the VDRL test result was still positive at a titre of $1 / 8$ and after seven months it was $1 / 4$. The serology test results of the child took the following course: after one week FTA-ABS positive, VDRL positive at a titre of $1 / 2$, and $19 \mathrm{~S}$ (IgM) FTA-ABS negative; five weeks later TPHA, FTA-ABS, and 19S (IgM) FTA-ABS weakly positive, VDRL negative; and at 12 weeks TPHA and FTA-ABS weakly positive, VDRL not undertaken, and 19S (IgM) FTAABS negative. The child had no clinical signs of congenital syphilis and was not treated. Unfortunately no further tests were performed and so no definite conclusion about the presence or absence of congenital syphilis was possible. In view of the negative VDRL result in week 6 and the negative result in the 19S (IgM) FTA-ABS test in week 12, however, infection seems unlikely.

Two women (cases 12 and 13) gave birth only one week after treatment for syphilis had started. The $19 \mathrm{~S}$ (IgM) FTA-ABS tests of the umbilical cord blood of both babies gave positive results. One baby (case 12) had clinical signs such as desquamation of the skin on the inside of the wrists and hepatosplenomegaly. The CSF of the other (case 13) showed an increase in protein concentration and a positive VDRL test result (1/4). Both children were treated with penicillin.

The fourth column of the table shows that the positive 19S (IgM) FTA-ABS test results were probably not related to congenital syphilis in four children. In two (cases 10 and 11 (see above)) this was possible, and in two (cases 9 (see above) and 14) it was certain. Case 14 concerned the child of a woman who had had secondary syphilis three years before pregnancy, when she had been treated with procaine penicillin $10.8 \mathrm{MIU}$. In week 18 of pregnancy she was given prophylactic treatment. The umbilical cord serum of the child gave positive results in the TPHA and the FTA-ABS test, but negative results in the VDRL and 19S (IgM) FTA-ABS tests. After seven weeks the antitreponemal IgM test gave a positive result but the other test results remained unchanged. After nine weeks the antitreponemal IgM test gave a negative result again. Serological tests were continued. After 23 weeks only the FTA-ABS gave a positive result, and after 38 weeks all tests gave negative results.

Column 5 of the table concerns five adopted children, one from Honduras, three from Sri Lanka, and one from Indonesia. One child presented with clinical symptoms, the others were screened on entry and were serologically monitored.

The parental characteristics we studied were: the type of obstetric care, age, civil status, gestation, and parity of 14 of the 19 mothers. The histories of the five mothers of the adopted children were not known. Six of the 14 mothers attended a gynaecologist for their pregnancy check up, seven attended a midwife, and the check up details of one mother were not known. This pattern was not significantly different from that of the total childbearing population. ${ }^{2} \mathrm{~A}$ gynaecologist atten- 
ded the deliveries of the two unscreened women (case 6 and 7); the two women who had not been treated despite positive syphilis serology results (cases 8 and 9) were attended by a midwife.

The ages at parturition of the mothers in the study group ranged between 17 and 38 ; most women were aged 25 to 29 . The difference between the study group and the total childbearing population was not significant. ${ }^{3}$ The civil status of the mothers during the pregnancies studied was as follows: seven were married to the fathers of the children, one cohabited with the father, two were divorced and had new partners, and three were unattached. As only $8 \%$ of the total number of births in The Netherlands in 1984 were extramarital, ${ }^{4}$ the difference from the incidence in study group was appreciable. Regarding the length of gestation and parity of the mothers in the study group, two had histories of immature birth, one of spontaneous abortion, and one of induced abortion. Three women were para 0 , eight ranged from para 1 to 5 , and the parity of the remaining three was unknown. The nationalities of the mothers were as follows: four were Dutch, four came from Surinam, two from the Cape Verde Islands, two from Turkey, one from Morocco, and one from Germany. For comparisons: in $19856 \%$ of all neonates in The Netherlands had mothers of foreign origin. ${ }^{5}$ Thus appreciably more foreign mothers delivered children with syphilis.

\section{Discussion}

An analysis of the 14 pregnancies yielded the following findings. Pregnancy check ups had been performed in 13 , but screening for syphilis in only 10 . The age of the mothers in the study group did not differ significantly from that of other mothers, but women of foreign origin and extramarital pregnancies were overrepresented. The sexual partners of four women (cases $1,4,7$, and 11) were treated for syphilis during their wives' pregnancies, but adequate contact tracing was omitted. This resulted in lack of treatment and hence in congenital syphilis in three children (cases 1, 4, and 7). Lack of adequate contact tracing therefore appears to be an important cause of the continued occurrence of congenital syphilis in The Netherlands. Doctors should remember that not only a first pregnancy but also subsequent pregnancies may lead to congenital syphilis because the mother may have become infected in the interim or a previous child may not have been infected even though the mother was infective. ${ }^{6}$ Generally, however, the risk of transmission of the infection from the mother depends on the stage of maternal infection: the more recent the infection, the graver the risk of congenital syphilis. ${ }^{7}$

Not screening for syphilis because of negative syphilis serology results in previous pregnancies must be regarded as an omission, as indicated by cases 6 and 7. Pregnant women with positive syphilis serology results merit special attention. Untreated syphilis in a pregnant woman-regardless of the stage of the infection - can lead to congenital syphilis in the child. Adequate treatment consists of penicillin courses that differ in duration, dosage, and mode of administration according to the stage of infection. The Dutch National Health Council recommends the same treatment for pregnant and non-pregnant women. ${ }^{8}$ Failure of courses of treatment with penicillin has been described in women given insufficient dosages, ${ }^{910}$ treated in the last three months of pregnancy, ${ }^{9}$ or who gave birth within two weeks of the start of treatment. ${ }^{10}$

In the event of hypersensitivity to penicillin, erythromycin may be given. Several studies, however, have shown that erythromycin does not always prevent congenital syphilis. ${ }^{11} 12$ When the mother has been treated for syphilis with erythromycin, the neonate requires extra attention. ${ }^{1213}$

One (case 10) mother was adequately treated for early as well as for late syphilis, but not for possible neurosyphilis. She refused a lumbar puncture, and neurosyphilis could therefore neither be diagnosed nor excluded. This woman may have been inadequately treated, but it seems more likely that the positive antitreponemal IgM test result did not indicate congenital syphilis. The same applies to another woman (case 11), though the risk of neurosyphilis seems far smaller because her partner had probably had early syphilis, as his VDRL test result was positive at a titre of $1 / 128$.

Apart from possibly false positive IgM test results (that is, positive IgM test results not indicating congenital syphilis) in two children (cases 10 and 11 ), two (cases 9 and 14) had unmistakably false positive antitreponemal IgM test results. Three of these four children (cases 9, 11, and 14) were born in 1982, when the 19S (IgM) FTA-ABS test was still being performed without prior absorption with a sorbent. Absorption was introduced in 1983, and the test has since been more specific. ${ }^{1314}$ Nevertheless, prior absorption also gives a chance of a false positive result. This may be because of technical errors or errors of judgement. Moreover, Pickering has stated that maternal blood with IgM antibodies can enter the fetal circulation by so-called transplacental transfusion. ${ }^{13}$ The half life of IgM antibodies is four to five days, and false positive test results can therefore only be observed in the first weeks after parturition. On the other hand a negative antitreponemal IgM test result does not exclude congenital syphilis because some time may pass before the antibody production in the child is sufficient. ${ }^{13}$ One baby in our study (case 1) had negative VDRL and Wasserman test results three weeks after birth, a weakly positive FTA-ABS test result three weeks later, 
and full blown positive results even in the 19S (IgM) FTA-ABS test at three months. Another child (case 3) also had a negative 19S (IgM) FTA-ABS test result at four weeks and a positive one at nine weeks.

In 1982-5 five cases of congenital syphilis were reported to the chief public health inspector of the Ministry of Health. By tracing the 19 babies with positive 19S (IgM) FTA-ABS test results, we found that at least 15 , and perhaps 17 , had the disease. Congenital syphilis therefore appears to be underreported. Strengthening of screening in early and late pregnancy in high risk groups, prompt treatment of women positive for syphilis, and adequate follow up are ways of helping to prevent congenital syphilis. Adequate contact tracing, starting with male index cases, also appears to be important. We only studied the failures of control of syphilis in pregnancy.

It is not known how many women are tested for syphilis during pregnancy and have a negative test result. As the generally accepted policy is to test for syphilis during pregnancy, every woman should be tested, regardless of her age, nationality, civil status, or history. The test should preferably be performed within the first three months of pregnancy, but must not be omitted if a pregnant women reports for a check up later in pregnancy. The test should be repeated in the last term of pregnancy in all women with an increased risk of contracting syphilis. Adequate treatment should be instituted in all pregnant women with indications of untreated or inadequately treated syphilis. The newborn child should be examined and submitted to serological tests to minimise the consequences of treatment failure. Monitoring the child should not be discontinued until all serological tests give negative results. Follow up of the child is advisable, particularly if the mother was treated late in the course of pregnancy or was treated with erythromycin.

We recommend that all children adopted from foreign countries are tested for syphilis on entry into The Netherlands to ensure that treatment, if required, can be started as soon as possible. Continuing this retrospective study might be useful for future adjustment of strategies to prevent congenital syphilis and other possible infections in mothers and children.

\section{References}

1 Murphy FK, Patamasucon P. Congenital syphilis. In: Holmes KK, Mårdh P-A, Sparling PF, Wiesner PJ, eds. Sexually transmitted diseases. New York: McGraw Hill, 1984;352-73.

2 Centraal Bureau voor de Statistiek. Statistisch zakboek. 'sGravenhage, The Netherlands: Staatsuitgeverij/CBS Publikaties, 1985:52.

3 Centraal Bureau voor de Statistiek. Statistisch zakboek. 'sGravenhage, The Netherlands: Staatsuitgeverij/CBS Publikaties, 1985:40.

4 Centraal Bureau voor de Statistiek. Maandstatistiek van de Bevolking, Februari 1986. 's-Gravenhage, The Netherlands: Staatsuitgeverij/CBS Publikaties, 1986:11.

5 Centraal Bureau voor de Statistiek. Maandstatistiek van de Bevolking, Februari 1986. 's-Gravenhage, The Netherlands: Staatsuitgeverij/CBS Publikaties, 1986:11-2.

6 Davies PA. Maternal and fetal infection. Clin Obstet Gynaecol 1974;1:17-39.

7 Fiumara NJ. When a pregnant woman has syphilis. Contemporary Obstetrics and Gynaecology 1981:17:75-99.

8 Dutch National Health Council. Advice concerning sexually transmitted diseases. 's-Gravenhage, The Netherlands: Staatsuitgeverij/CBS Publikaties, 1986:176-7.

9 Mascola L, Pelosi R, Alexander CE. Inadequate treatment of syphilis in pregnancy. Am J Obstet Gynecol 1984;150:945-7.

10 Schofield CBS. Sexually transmitted disease surveillance. Br Med J 1982;284:825

11 Hartmann JF, Lescoeur B, Mercier JC, et al. Syphilis neo-natal malgré le traitement de la mère par l'érythromycine. Presse Med 1984;13:2133-5.

12 Hashisaki P, Wertzberger GG, Conrad GL. Erythromycin failure in the treatment of syphilis in a pregnant woman. Sex Transm Dis 1983;10:36-8.

13 Pickering LK. Diagnosis and therapy of patients with congenital and primary syphilis. Pediatr Infect Dis 1985;4:602-5.

14 Muller F. Der 19S-IgM-FTA-ABS-test in der Serodiagnostik der Syphilis. Immun Infekt 1982;10:23-34. 